\title{
APPLICATION OF BOOLEAN ALGEBRA TO PROVING CONSISTENCY AND INDEPENDENCE OF POSTULATES*
}

\section{BY B. A. BERNSTEIN}

1. Object. The usual procedure in proving the consistency of a set of postulates is to find such an interpretation of the undefined ideas involved in the postulates as to make all the postulates true propositions. The procedure in proving the independence of a particular postulate from the rest of the set is to find such an interpretation of the undefined ideas as to make that postulate, and only that one, false. But there exists no method of finding these interpretations. The object of this paper is to show how Boolean algebra partially provides such a method.

2. General Procedure. The postulates of a mathematical science are conditions on the classes, operations, and relations constituting the undefined ideas of the science. There is an infinitude of Boolean algebras. $\dagger$ In each of these algebras there is, for every positive integer $n$, the general $n$-ary operation

$$
A x y \cdots t+B x y \cdots t^{\prime}+\cdots+L x^{\prime} y^{\prime} \cdots t^{\prime} \ddagger
$$

and the general $n$-adic relation

$$
A x y \cdots t+B x y \cdots t^{\prime}+\cdots+L x^{\prime} y^{\prime} \cdots t^{\prime}=0 .
$$

The procedure constituting a partial method of proving the consistency and the independence of a set of postulates consists, in the case of a consistency proof, in obtaining the conditions on the discriminants of (I) and (II) which will make (I) and (II) satisfy all the given postulates; if these Boolean conditions are consistent, $\S$ the given postulates are consistent. In the case of

* Presented to the Society, June 20, 1929.

$\dagger$ There is a Boolean algebra the cardinal number of whose elements is that of the continuum; one whose elements are denumerably infinite; and one for every positive integer $n$, the number of whose elements is $2^{n}$.

$\ddagger$ The usual Boolean notation is used, with $a^{\prime}$ denoting the negative of $a$.

$\S$ The determination of the consistency of a set of Boolean relations is, as is well known, always possible. The vanishing of the product of the discriminants of the single relation equivalent to the set is the necessary and sufficient condition for this consistency. 
proof of the independence of a postulate $P$ from the other postulates, the procedure consists in obtaining the conditions on the discriminants of (I) and (II) which will make (I) and (II) contradict $P$ and not contradict any of the other postulates; if these Boolean conditions are consistent, postulate $P$ is independent of the other postulates. If in the case of a consistency proof the resulting Boolean conditions are inconsistent, the consistency of the postulates remains undetermined. Similarly for the case of an independence proof. It is this possibility of an inconsistent set of resulting Boolean conditions which makes my procedure only a partial method for determining consistency and independence.

It is to be observed that the method here outlined does not require the actual finding of the desired Boolean consistency and independence systems, though such systems, when the Boolean conditions are consistent, can always be found.* The consistency of the resulting Boolean conditions proves that a desired Boolean proof-system can be found, and this possibility is sufficient for our purpose.

It is to be observed also that the conditions on the discriminants of (I) and (II) corresponding to a given postulate may be used in the investigation of consistency and independence of any set of propositions involving that postulate. At the same time, these conditions give valuable information concerning the operations and relations of Boolean algebra.

The proposed procedure of investigating consistency and independence of postulates thus gives results that are generally of far wider application than the results obtained by the usual methodless procedure.

The method outlined above will be made clearer by applying it to a set of postulates for abelian groups and to a set of postulates for series.

* The desired proof-system is simply the system in which the elements are the elements of the Boolean algebra employed, and the operations and relations are the operations and relations of forms (I) and (II) with the discriminants satisfying the conditions in question. Thus, the Boolean consistency system for postulates $P_{1}-P_{3}$ of $\S 3$ below, is the system $(K, 0)$ in which $K$ is the class of elements of any Boolean algebra and $a \circ b$ is the operation $(\alpha)$ with the discriminants satisfying condition (4). 
3. Application to Postulates for Abelian Groups. An abelian group is a class $K$ containing a binary operation $O$ satisfying postulates $P_{1}-P_{3}$ following:*

$P_{1} . a \circ(b \circ c)=(a \circ b) \circ c$, whenever the indicated elements belong to $K$.

$P_{2}$. For any two elements $a, b$ of $K$ there is an element $x$ of $K$ such that $a \circ x=b$.

$P_{3} . a \circ b=b \circ a$, whenever the indicated elements belong to $K$.

To investigate the consistency and the independence of $P_{1}-P_{3}$, consider the general binary Boolean operation

$$
A a b+B a b^{\prime}+C a^{\prime} b+D a^{\prime} b^{\prime} .
$$

The necessary and sufficient conditions that $(\alpha)$ satisfy $P_{1}, P_{2}$, $P_{3}$ are, respectively: $\dagger$

$$
\begin{gathered}
D=A D, \quad\left(B C^{\prime}+B^{\prime} C\right)\left(A D+A^{\prime} D^{\prime}\right)=0, \\
B=A^{\prime}, C=D^{\prime}, \\
B=C .
\end{gathered}
$$

From (1) - (3), it is seen that $(\alpha)$ will contradict $P_{1}, P_{2}, P_{3}$ if, respectively,

$$
\begin{gathered}
D A^{\prime}=1, \\
A B+A^{\prime} B^{\prime}=1, \\
B C^{\prime}+B^{\prime} C=1 .
\end{gathered}
$$

Relations (1) - (3) are together equivalent to the relation

(4) $f(A, B, C, D) \equiv D A^{\prime}+A B+A^{\prime} B^{\prime}$

$$
+C D+C^{\prime} D^{\prime}+B C^{\prime}+B^{\prime} C=0 \text {. }
$$

Relations (1)', (2), (3) are together equivalent to

$$
f_{1}(A, B, C, D) \equiv 1=0 .
$$

Relations (1), (2)', (3) together are equivalent to

(6) $f_{2}(A, B, C, D) \equiv D A^{\prime}+A B^{\prime}+A^{\prime} B+B C^{\prime}+B^{\prime} C=0$.

* See Transactions of this Society, vol. 4 (1903), p. 27.

† See Transactions of this Society, vol. 26 (1924), pp. 171-173. 
Finally, relations (1), (2), (3)' together are equivalent to

$$
f_{3}(A, B, C, D) \equiv A^{\prime}+B+C^{\prime}+D=0 .
$$

Since the discriminant $f(0,1,1,0)$ vanishes, condition (4) is possible. Hence, postulates $P_{1}-P_{3}$ are consistent.

Condition (5) is impossible, and the question of the independence of postulate $P_{1}$ remains unsettled.*

Since $f_{2}(1,1,1,1)=0$, condition (6) is possible. Hence $P_{2}$ is independent of the other postulates.

Finally, since $f_{3}(1,0,1,0)=0$, condition ( 7$)$ is possible. Hence, $P_{3}$ is independent of the other postulates.

4. Application to Postulates for Series. $\dagger$ The elements $a, b$, $c, \cdots$ of a class $K$ form a series with respect to a dyadic relation $S$, if they satisfy the postulates $Q_{1}-Q_{3}$ following:

$Q_{1}$. If $a \neq b$, then either $a S b$ or $b S a$.

$Q_{2}$. If $a S b$, then $a \neq b$.

$Q_{3}$. If $a S b$ and $b S c$, then $a S c$.

Consider the general dyadic Boolean relation

$$
A a b+B a b^{\prime}+C a^{\prime} b+D a^{\prime} b^{\prime}=0 .
$$

This relation, I have shown, can be a serial relation only if the Boolean algebra is a two-element one. Let $(\beta)$, then, be the general dyadic relation in a two-element Boolean algebra.

The necessary and sufficient conditions that $(\beta)$ satisfy $Q_{1}$ and $Q_{2}$ are, respectively:

$$
\begin{aligned}
& B C=0, \\
& A D=1 .
\end{aligned}
$$

The necessary and sufficient condition that $(\beta)$ satisfy $Q_{\mathbf{z}}$ nonvacuously is

$$
(A+D) B^{\prime} C^{\prime}=0, A D=0 .
$$

The necessary and sufficient condition that $(\beta)$ satisfy $Q_{3}$ vacuously is

$$
A D(B+C)=1 .
$$

* That the denial of (1) is inconsistent with (2) and (3) may also be seen by observing that (2) and (3) imply (1).

$\dagger$ For the results through condition (iv) below, see this Bulletin, vol. 32 (1926), pp. 523-524. 
From (i)-(iii), relation $(\beta)$ will contradict $Q_{1}, Q_{2}, Q_{3}$ if, respectively:

$$
\begin{aligned}
& B C=1, \\
& A D=0, \\
& A D=1 .
\end{aligned}
$$

Conditions (i)-(iii) are together equivalent to

$$
1=0 \text {. }
$$

Conditions (i), (ii), (iv) are together equivalent to

$$
F(A, B, C, D) \equiv B C+A^{\prime}+D^{\prime}+B^{\prime} C^{\prime}=0 .
$$

Conditions (i)', (ii), (iv) are together equivalent to

$$
F_{1}(A, B, C, D) \equiv B^{\prime}+C^{\prime}+A^{\prime}+D^{\prime}=0 .
$$

Conditions (i), (ii)', (iii) are together equivalent to

$$
\text { (viii) } F_{2}(A, B, C, D) \equiv B C+A D+(A+D) B^{\prime} C^{\prime}=0 \text {. }^{*}
$$

Finally, conditions (i), (ii), (iii)' are together equivalent to

$$
F_{3}(A, B, C, D) \equiv B C+A^{\prime}+D^{\prime}=0 .
$$

Relation ( $v$ ) is impossible. Hence, in a two-element Boolean algebra there is no relation $(\beta)$ which satisfies $Q_{1}-Q_{3}$ nonvacuously, and the question of the consistency of $Q_{1}-Q_{3}$ remains unsettled.

But relation (vi) is possible, since the discriminant $F(1,1,0,1)$ vanishes. Hence, postulates $Q_{1}-Q_{3}$ are consistent.

Relation (vii) is possible, since $F_{1}(1,1,1,1)=0$. Hence postulate $Q_{1}$ is independent of the other postulates.

Relation (viii) is possible, since $F_{2}(0,0,0,0)=0$. Hence $Q_{2}$ is independent of the other postulates.

Finally, relation (ix) is possible, since $F_{3}(1,0,0,1)=0$. Hence, $Q_{3}$ is independent of the other postulates.

The University of California

* If (iv) had been used here instead of (iii), the resulting condition would have been $1=0$, from which no conclusion could have been drawn with regard to the independence of $Q_{2}$. 